\title{
Mass-analyzed Threshold Ionization Spectrometry with Scrambling Field Optimized for the Study of State-selective Ion Reaction Dynamics ${ }^{*}$
}

\author{
Sang Tae Park, Hong Lac Kim, ${ }^{\ddagger}$ and Mỵung Soo Kim \\ National Creative Research hitiative for Conmol of Reaction Dynamics and School of Chemistry. \\ Seoul tational linversity. Seoul 151-7+2. Sorea \\ Received February 25. 2002
}

\begin{abstract}
Mass-analyzed threshold ionization (MATl) technique is optimized to generate substantial amount of stateselected molecular ions sufficient for dynamics study. The main strategy is to stabilize intermediate $(n=\mathrm{I}(0)$ 200) Ryddberg states by $/ m$-mixing induced by AC field. Electrical jitter inherent in high voltage switching is utilized for this purpose. A related teclmique to locate the MATl onset is also described.
\end{abstract}

Key Words : Mass-analyzed 1hreshold ionization

\section{Introduction}

State selection ${ }^{1,2}$ of gas phase molecules is the usual starting point for the study of intramolecular and reaction dynamics. ${ }^{3}$ reaction control. ${ }^{4-5}$ and other manipulation of such systems. Combination of supersonic expansion ${ }^{7}$ and laser techniques is useful for preparation of state-selected neutrals. State selection is much more difficult for gas phase molecular ions. Conventional ionization techniques such as electron ionization and photoionization generate molecular ions with a wide distribution of quantum states. "Situation is not much better with more elaborate schemes such as resonance-enhanced multiphoton ionization. Injecting ions generated by conventional means into supersonic jet often helps state selection. ${ }^{7}$ This techuique is not generally applicable. however. due to various complications involved. Hence. one usually has to be content with energy selection rather than state selection in ionic studies using techniques such as photoelectron-photoion coincidence ${ }^{\text {li }}$ and charge exchange ionization. $^{11}$

Zero electron kinetic energy (ZEKE) spectroscopy ${ }^{12}$ is a useful technique to obtain accurate thermochemical and spectroscopic information on molecular cations. In this techuique. molecule is excited to a Rydberg state which lies close to the ionization threshold and ionized by electric field pulse. ${ }^{13}$ Lifetime of a Rydberg state gets longer as its energy approaches the ionization threshold. ${ }^{1+}$ Hence. by using a long delay time (on the order of $10 \mu \mathrm{sec}$ ) between the Rydberg state formation and pulsed field ionization (PFI), ionization can be limited to Rydberg states lying very close to the ionization threshold. An additional advantage of using a very long delay time is that electrons generated by direct ionization can be effectively eliminated during this time. Since all the rovibrational states of a cation have the

\footnotetext{
l' 'his paper is dedicated to l'fofessor Sang Chul Shim for his distinguished achievements in chemistry

t]'emanent address: 1 Jepartment of C']emistry; Kangwon National l hnversity, Chuncheor 200-70) Korea

"To whom correspondenee should be address. Phonc: +82-2-8806652. Fax: +82-2-889-1568, E-mail: myungsoocaplaza.snu.ac. br
}

associated series of Rydberg states. a high resolution spectrum of the cation can be obtained by scanning the excitation wavelength and detecting electrons generated by PFI.

When one detects molecular ions instead of electrons in ZEKE. the technique begins to have relevance to state selection of molecular ions. Feasibility of this technique which is called mass-analy zed threshold ionization ${ }^{15}$ (MATI) was demonstrated some years ago. Currently. MATI is used as a technique complementary to ZEKE. its recognized advantage being the capability to identify the ionic species responsible for the ZEKE electron generated. Namely, its capability to generate state-selected molecular ions has not been utilized until very recently..$^{16}$ As in ZEKE, molecular ions generated by direct photoionization together with Rydberg neutrals must be eliminated before pulsed field ionization of the latters in MATI. Coulomb repulsion and timy stray electric field present in the instrument are sufficient for this purpose in ZEKE. In contrast. renıval of heavy molecular ions in MATI is a more difficult task. Either a very long delay time has to be used or all extra electric field. so-called spoil field. has to be applied to separate ions generated by direct photoionization from the Rydberg neutrals. However, application of the spoil field ionizes neutrals in states very close to the ionization threshold. Exciting neutrals to Rydberg states well below the threshold is not helpful either because lifetime of a Rydberg state shortens quickly as its energy lowers. Allother difficulty in MATI teclunique arises from the need to use high voltage switching electronics for ioll acceleration in contrast with low voltage electronics used in ZEKE. Presence of stray field is unavoidable in the former. This again results in field ionization of Rydberg neutrals close to the threshold. Overall effect is the deterioration in spectral resolution and signal intensity: Overcoming these difficulties is essential for generation of state-selected ion bean with sufficient intensity for dynamics study.

In this paper. we report a method to lengthen the lifetime of Rydberg neutrals significantly and hence enthance the MATI signal intensity also by applying a weak AC field. or 
scrambling ficld. at the time of laser irradiation. A method to locate the MATJ onset will be presented also.

\section{Experimental Section}

Nitric oxide. fluoroben/ene. and cyclopentene were purchased from Aldrich and benzene from Merck and used withoul further purification. Nitric oxide was premixed with $\mathrm{He}$ in a gas rescrvoir while liquid samples were secded in $\mathrm{He}$ carrier gas at room temperature. They were expanded into a source chamber through a nozzle orifice (0.3 mum diameter. General Valve) and skimmed through a 1 mm-diameter skimmer (Bcam Dynamics) to enter a differentially pumped ionization chamber. The backing pressure was -2 atm typically and background pressure of the ionization chamber was maintained below $10^{-7}$ torr.

Details of the experimental setup. Figure l. were described previously. ${ }^{16.17}$ The method to generate coherent vacuum ultraviolet (VUV) radiation by four-wave difference frequency mixing ${ }^{l \%}$ in $\mathrm{Kr}$ gas is as follows. The second harmonic output of a Nd: YAG laser (Continuum PL8000. 5 ns) was used to pump a dye laser (Continuum NDG000) to generate $638 \mathrm{~nm}$ laser pulses. Then it was Irequency-tripled to produce the $212.4 \mathrm{~nm}$ oulput $(-0.5 \mathrm{~mJ} /$ pulsc) which was used to excite the $\mathrm{Kr} 5 \mathrm{pl} / 1 / 2 \mathrm{p}_{-}-4 \mathrm{p}^{6}$ transition via two-photon resonant absorption. A laser pulse in the $500-540 \mathrm{~nm}$ range was also generated by a second dye laser pumped by the 355 nm oulput of another Nd: YAG laser. Two laser pulses were

\section{(a) top view}
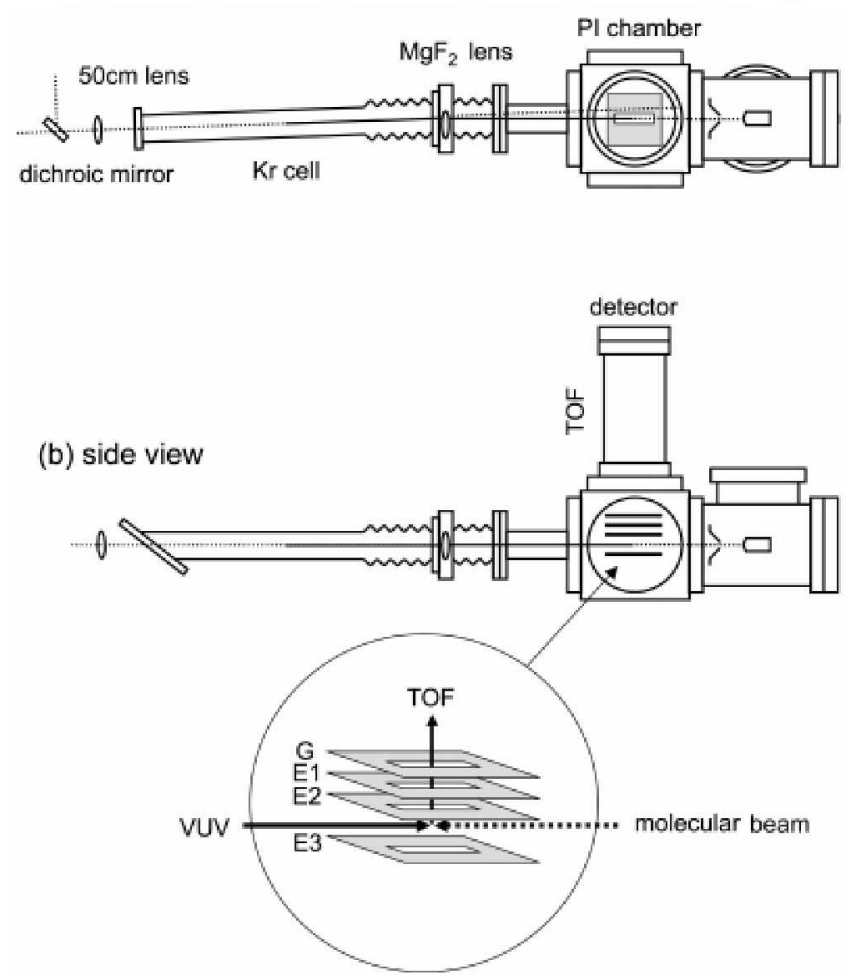

Figure 1. Schematics of VUV-MAII instrument. Sow lext for details.
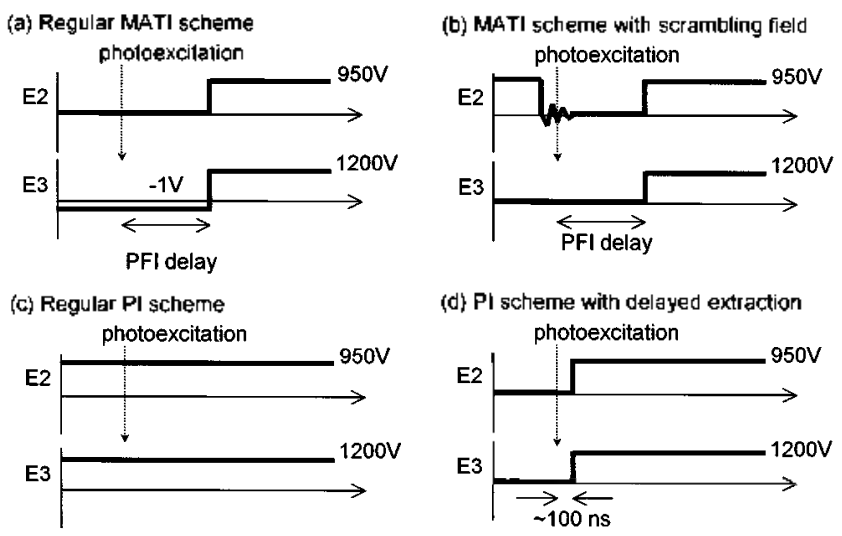

Figure 2. Voltage pulsing schemes. (a) Regular MATI scheme. (b) MAll scheme utilizing electronic jiller in high volkage switching as scrambling licld. (c) Regular PI scheme. (d) PI scheme with delased extraction. See text tor details.

then combined and loosely focused by a fused-silica lens (50) $\mathrm{mm}$ focal length) prior to entering the $\mathrm{Kr}$ gas cell. A $\mathrm{MgF}_{2}$ lens (250 $\mathrm{mm}$ nominal focal length) was placed offcenter at the exil of the Kr gas cell to separate the VUV laser output from the input laser pulses in the laser-molccular bcam intcraction region. ${ }^{19}$ The VUV laser in the 132 135 $\mathrm{nm}$ region was generated with the $\mathrm{Kr}$ pressurc optimized in the $\mathrm{l}-\mathrm{l}$ () torr range.

The VUV laser pulse was collincarly overlapped with the molccular bcam in a counter-propagation manner to maximizc the lascr-molccular bcam intcraction volume. ${ }^{23 !}$ Instead of the usual circular apcrturc. $+\mathrm{mm} \times 50 \mathrm{~mm}$ size slit electrodes were used to enhance ion collection efficiency. Figure 1. When needed. spoil ficld of $\sim 0.5 \mathrm{Vcm}^{-1}$ was applicd in the ionization region to remove ions produced by direct photoionization. To achicse pulsed-ficld ionization (PFI) of neutrals in the high Rydberg states. an electric ficld of $\sim 125 \mathrm{Vcm}^{-1}$ was applicd with the ficld direction perpendicular to that of the molecular and laser beans. Voltage and timing sequences are shown in Figure 2. which will be explained later. Ions generated were accelerated. flied through a field-free region. and were detected by a dual microchanuel plate (MCP) detector. MATI signal detected by MCP was preamplified and $\mathrm{A} / \mathrm{D}$ converted by a digital storage oscilloscope (LeCroy. LC33+AM). Either the full time-of-flight mass spectrum or selected regions of the spectrum as needed were transferred to a personal coniputer in real time.

\section{Results and Discussion}

ZEKE states. The energies $E_{\text {nim }}$ of a series of Rydberg states are described by the Rydberg formula.

$$
F_{n m i}=-\frac{R_{\mathrm{Mr}}}{\left(n-\delta_{l}\right)^{2}}
$$

Here $n . l$, and $m$ are the usual quantum numbers for H-like atoms. $R_{i / t}$ is the mass-dependent Rydberg constant $\left(R_{\infty}=\right.$ $\left.109737 \mathrm{~cm}^{-1}\right)$, and $\delta_{l}$ is the $/$-dependent quantum defect. A 
Rydberg state with the $n$ quantum number of 200 lics $-3 \mathrm{~cm}^{-1}$ below the corresponding series (ioni/ation) limit.

Lifetime of a Rydberg state has becn a subject of great interest. ${ }^{13.14 .21-39}$ Extrapolation of experimental lifetimes for lower $n$ states ${ }^{13}$ shows that $n \sim 200$ p-orbital Rydberg states of $\mathrm{NO}$ are expected to have lifetime of approximately $100 \mathrm{~ns}$. Then. a much longer lifetime observed experimentally ${ }^{21}$ which renders feasibility to the ZEKE and MATl 1echniques. is a remarkable phenomenon itself. $\mathrm{l}$ is generally thought that decay rate of a Rydberg state is dependent on the interaction between the Rydberg electron and the ionic core. Then. the lifetime of a Rydberg state would increase with its / quantum number because an electron in a high / orbital has small probability to be close to the ionic core. Unless built with extreme carc an apparatus tends to have a small stray ficld (a few tens of $\mathrm{mVcm}^{-1}$ ). ${ }^{22}$ This stray field would cause Stark spliting of the Rydberg state prepared and / is no longer a good quantum number. ${ }^{14.23}$ It is generally accepted that the / mixing induced by the stray ficld allows transitions from the low / states to the high / states and lengthens the lifetime approximately by $n$. ${ }^{14}$ For a Rydberg state with $n=$ 200 taken as an example above. the lifetime can become as long as $20 \mu \mathrm{s}$. in qualitative agreement with the cxperimental observations. The term ZEKE state has becn coined to differentiate the high $n$ and $/$ states expected to be generated under the actual ZEKE experimental condition from the high $n$-low / Rydberg states initially prepared by photocxcitation. ${ }^{12}$

It is not that presence of electric field has only benelicial effect of lenglhening the lifetime of Rydberg states. In the presence of an cxternal field $l \cdot$ (in $\mathrm{Vem}^{-1}$ ). the ionization limil of a Rydberg series is lowered from the field-frec limil by

$$
\Delta I\left(\mathrm{~cm}^{-1}\right) \sim 4 \sqrt{l} .
$$

For cxample. a stray ficld of $20 \mathrm{mVcm}^{-1}$ lowers the ionization threshold by $0.6 \mathrm{~cm}^{-1}$. Since low Rydberg states undergo rapid internal relaxation while those very close to the ionization threshold are field-ionized by the stray field. only those Rydberg states lying somewhat below the threshold, with $n=200-300$. will contribute to the ZEKE signal measured at $1-10 \mu$ s time delay after photoexcitation. With the presence of stronger electric field under the MATI condition. prompt ionization of high $n$ states is unavoidable while the low $n$ states undergo rapid internal decay. Hence, converting Rydberg states with $n$ quantum numbers in the intermediate range, $n=100$-200, to the high $/$ ZEKE states becomes a prerequisite for a successful MATI experiment.

Lifetime lengthening by scrambling field. As has been pointed out earlier. a weak DC field contributes to $/$ mixing but does not induce any $m$ mixing. On the other hand. inhomogeneous electric field is known to induce both $l$ mixing and $m$ mixing. ${ }^{14.27 .28}$ The latter enhances the nonpenetrating character of the Ryddberg orbital in ligh Rydberg states making the states extremely insensitive to decay processes such as autoionization. predissociation. and intramolecular relaxation. Effect of inhomogeneous field is especially important in the absence of DC ficld which splits Rydberg states by Stark effect. Such an inhomogencous clectric field is produced by particles. especially charged particles. located near the Rydberg neutrals. Alternatively. inhomogeneous ficld can be applicd intentionally in the form of a pulse or AC field. We will call it the scrambling licld. Gencral finding in such cxperiments is the broadening of a ZEKE peak toward the low $n$ quantum number direction. indicating that lifetime of lower Rydberg states is lengthened due to the scrambling lield. Namely. lengthening the lifetime of Rydberg neutrals in the intermediate $n$ quantum states is not benclicial to the ZEKE spectroscopy because a wide range of Rydberg neutrals would then survive during the delay time and contributc to the PFI signal. resulting in poor spectral resolution. In MATl spectroscopy where high n Rydberg neutrals are ficld-ionized by spoil ficld. however. lengthening the lifetime of Rydberg neutrals in the intemediate $n$ quantum states would help to maintain the PFI signal level.

Schematic drawing of the electrode assembly used in this work to study the elfect of scrambling ficld on the lifetime of Rydberg neutrals is shown in Figure 1 . The assembly consists of threc clectrodes $E_{1} . E_{2}$ and $E_{3}$. of which $E_{1}$ is the ground clectrode. Usual pulsing scheme. or scheme I. is shown in Figure 2(a). Small DC voltage on the $E_{3}$ clectrode provides the spoil licld necded to remove direct ions. After some lime delay. high voltages are applicd on the $E_{2}$ and $E_{3}$ clectrodes for pulsed licld ionization of Rydberg neutrals and acceleration of MATI ions thus generated. High voltages on both clectrodes are necded for mass-independent time locusing of MATI signals. Unlike in ZEKE where only low voltage pulses are necded. high voltage switching necded in MATI makes it difficult to apply scrambling ficld between the $E_{2}$ and $E_{3}$ clectrodes. One may give up time focusing and pulses the $E_{3}$ voltage while the $E_{2}$ clectrode is used for application of the scrambling ficld. Then. the result is a very poor mass spectral resolution for MATI signals. To avoid the above difficultics. we derised a new scheme which utilizes the ringing. or jitter, present when a high voltage is switched off. In the scheme shown in Figure 2(b). or scheme II. the high voltage on the $E_{2}$ electrode is switched off just before laser irradiation. The voltage ringing with the frequency of $\sim 100 \mathrm{MHz}$ provides the scrambling field. With the scrambling field on. direct ions are renoved more rapidly than in the absence of this field. We attribute this to the DC component of scrambling field. which serves as the spoil field. By comparing with the measured disappearance rate of direct ions in the presence of externally applied spoil field. we estimate that the DC component of scrambling field is $\sim 0.3$ $\mathrm{Vcm}^{-1}$. Namely. the voltage ringing serves dual purposes of providing scrambling and spoil fields. Additional spoil field can be applied also in this schente but is usually not needed.

MATI experiment was performed for NO with schenles I and II. PFI delay time was varied to observe the effective lifetime of Rydberg neutrals. Time-dependent decays of MATI signals obtained with schemes I and II are shown in Figures 3(a) and 3(b), respectively: It is to be noted that most of the MATI signal decars rapidly. within $\sim 100 \mathrm{nsec}$, in 

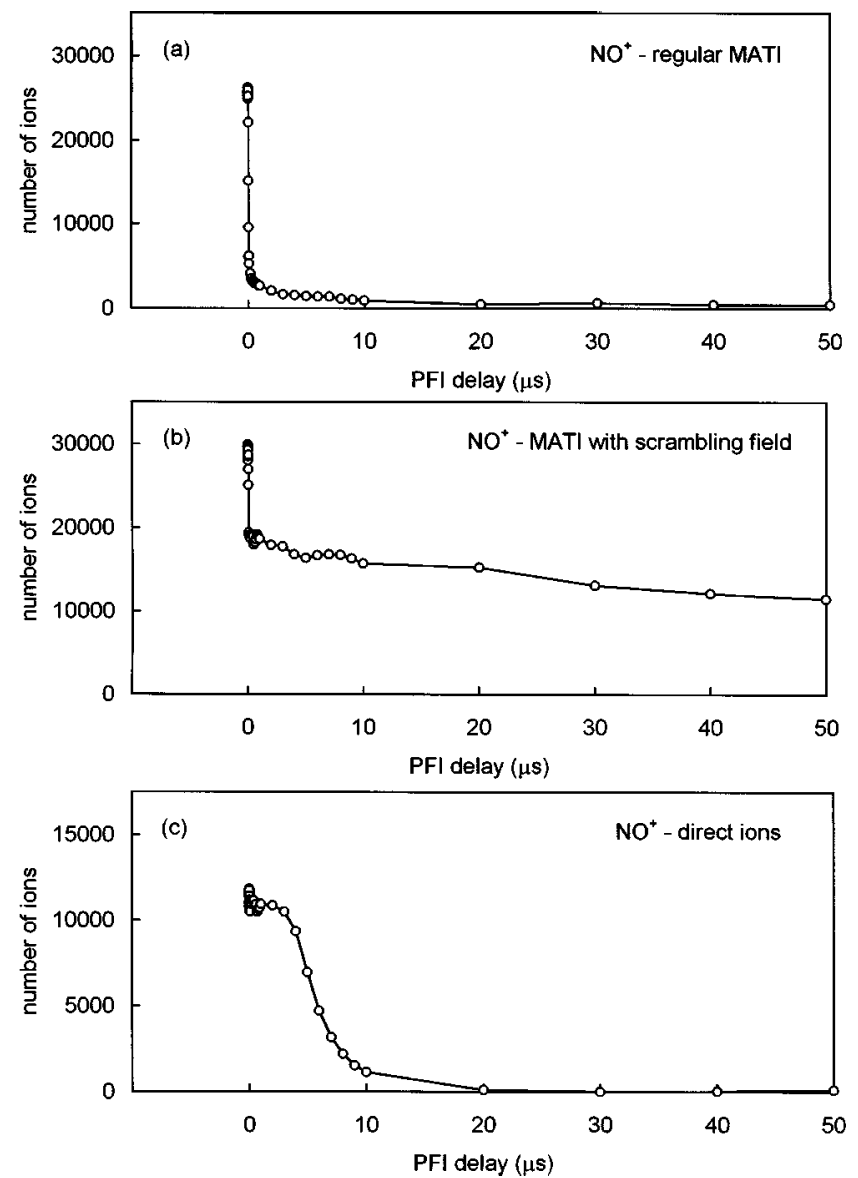

Figure 3. Ion vield as a lunction of delay lime between photoexcitation and pulsed field ionization of NO. (a) Regular MATT. (b) MATI with scrambling field. (c) Ions generated by direct photoionization.

scheme l. Even though similar rapid decay is observed in scheme II also. around $40 \%$ of the signal persists cien alicr $50 \mu \mathrm{sec}$ time delay. Also shown in the figure. Figure 3(c). is the time dependent decay of the direct ion signal obtained with scheme II with a laser wavelength where MATI signal was absent. It takes around $20 \mu \mathrm{sec}$ to remove the direct ions completely. Then. by using delay time longer than $20 \mu \mathrm{sec}$. a high resolution time-of-flight MATI spectrum with high intensity can be obtained which is free from direct ion contamination. Similar experments have been done for benzene. Figure 4 . Here again. lifetime enhancement by the scrambling field is apparent. We have carried out similar experiments for many other systems and observed comparable results. We could generate more than 500 state-selected molecular ions per laser pulse, which was sufficient for dynamics study:

Determination of the MATI threshold. As has been mentioned so far. the main difficultry in generating substantial amount of state-selected molecular ions by MATI is associated with the removal of direct ions and with the use of high voltage switching electronics. Our new scheme avoids this difficulty by lengthening the lifetime of lower-lying $(n=$ 100-200) Rydberg states utilizing high voltage jitter. Then. the energy levels of the Rydberg states sampled are quite
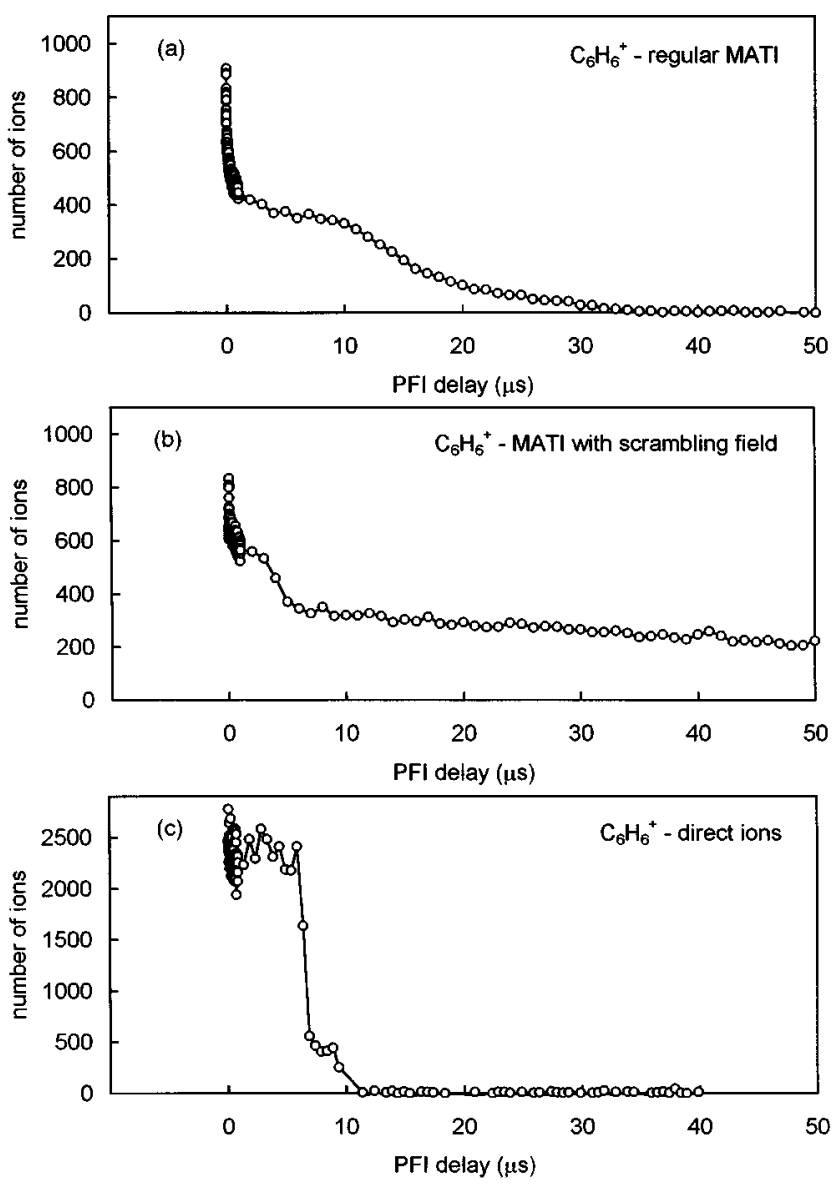

Figure 4. Ion yicld as a function of delay lime between photoexcitation and pulsed field ionization of benzene. (a) Regular MATI. (b) MATT with scrambling field. (c) Ions generated by direct photoionizalion.

below the ionization threshold. Scarching for a MATI pcak. usually the $0-0$ peak. becomes a practical difficulty in this scheme. which requires substantial amount of time and effort.

A simple method to find the MATI onset. or the $0-0$ peak position. is to record the photoionization efficiency (PIE) curve. or the intensity of the direct ion current as a function of the photon energy: In the usual schene to record the PIE curve. high $\mathrm{DC}$ voltages are applied to the electrodes $E_{2}$ and $E_{3}$ to extract direct ions generated by photoionization between these electrodes. Figure 2(c). The PIE curve of benzene thus obtained is compared with the $0-0$ MATI peak position in Figure 5(a). It is to be noted that the PI threshold lies substantially below the MATI onset and is not helpful in locating the latter. There are two reasols for lowering of the PI threshold. First, due to the presence of high electric field in the ionization region, the ionization thresholds of lowerlying Rydberg states are lowered. Or. the Rydberg states lying well below the ionization threshold are field-ionized under this condition. Second is the ionization due to hot band transition. whose contribution varies with the experinental condition such as the molecular beam expansion condition. To avoid the first difficulty we attempted a switching 

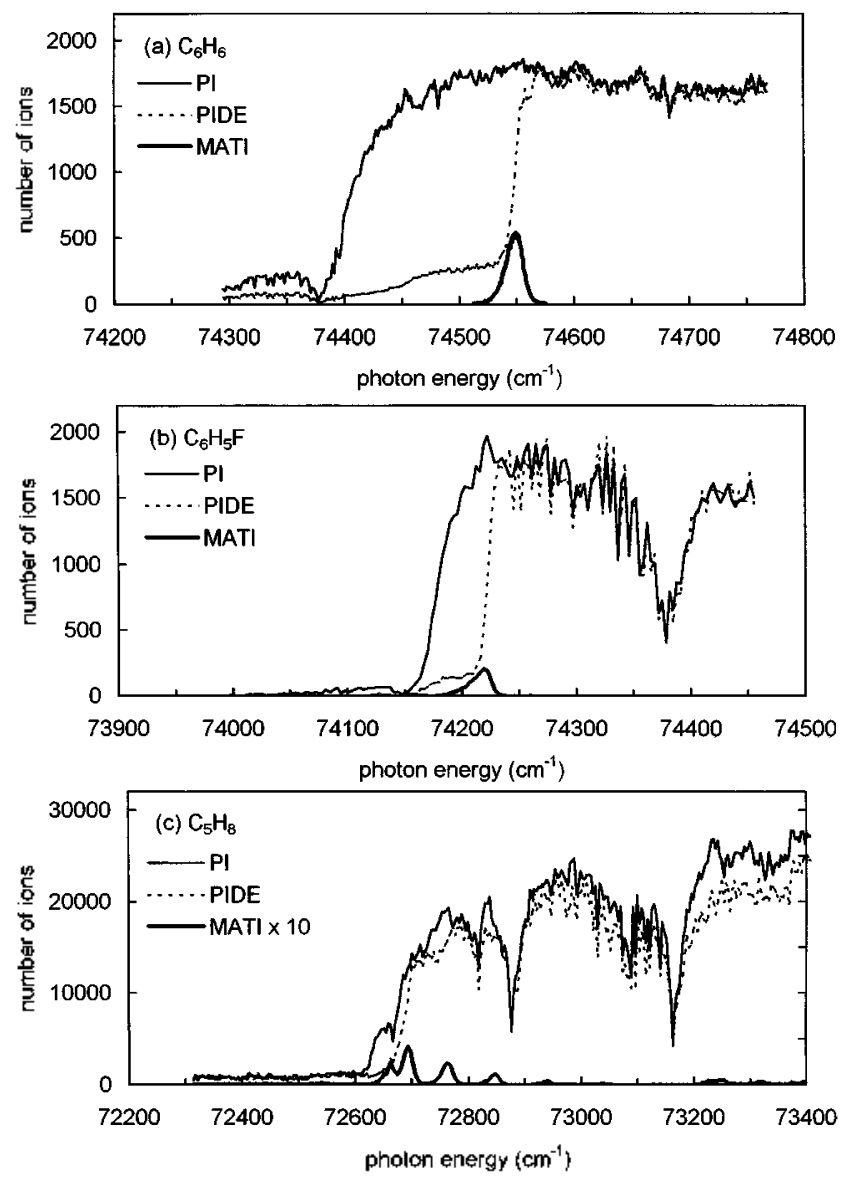

Figure 5. Photoionization elificiancy curies oblained under regular (PI) and delaved extraction (PIDF) conditions are compared with MATI spectra for (a) benzene, (b) thorobentene, and (c) cyclopenlens.

scheme for extraction of the direct ions. In this scheme. which will be called photoionization with delaycd extraction (PIDE), photoionization is carried out under the field-free condition. Then. after $\sim 100$ ns time delay, the accelerating field is switched on. Figure 2(d). PIE curve of benzene obtained with the PIDE teclunique is shown in Figure 5(a) also. It is to be noted that the PIE curve recorded with PIDE is shifted toward higher energy compared to the one recorded under the DC condition. It is because the lowerlying Rydberg neutrals decayed during the 100 nsec delay and did not undergo field-ionization anymore when high electric field was switched on. Even though photoionization by hot band transition also appears under the delared extraction. the correct ionization threshold can be easily identified. More importantly: the figure shows that the PI threshold under the delayed extraction condition nearly coincides with the MATI onset. Namely by recording PIE curve under the delayed extraction condition. the MATI onset can be readily located. We perfonmed similar experiments for various molecules and observed good coincidence between the PIDE and MATI onsets. The results for fluorobenzene and crclopentene are shown in Figures 5(b) and 5(c), respectively. as examples. It is to be noted that the mismatch between the regular PI and MATI onsets differs for different systems and also for different experimental conditions. Regardless. the MATI onset is always coincident with the Pl onset observed under the delaycd cxtraction condition.

\section{Conclusion}

Electronic jitter appearing when a high roltage is switched off has been utilized as a means to apply $\mathrm{AC}$ ficld. or scrambling field. to high Rydberg neutrals generated by resonant VUV cxcitation, lt has been found that strong MATI signal persists alter PFI delay time as long as $50 \mu \mathrm{sec}$. It is thought that the lifetime of Rydberg neutrals somewhat below the ionization threshold $n=100-200$. has been lengthened through the $/ m$-mixing induced by scrambling licld. Most importantly, the intensity of the state-selected molccular ion bean gencrated by MATI has increased tremendously with the use of scrambling ficld. Now the technique scems to be adequate for the study of statesclective ion reaction dynamics.

Acknowledgment. This work was supported financially by CRI. the Ministry of Science and Technology. Republic of Korca. S. T. Park thanks the Ministry of Education. Republic of Korca. for the financial support through the Brain Korca 2l program.

\section{References}

1. NATO . ASI Series. Series (: Mathematical and Physical Sciences. vol. 245. Selectivity in Chemical Reactions: Whitehead. T. C.. Iids:- Kluner Academic Publishers: Ixordrecht. Netherlands 1988.

2. Hode Selective (hemistm Jortner I.: I evine. R. I): Pullman. 13. I ids: Kluwer Academic Publishers: Dordrecht. Netherlands. I991.

3. Levinc. R. D.: Bernstein. R. B. Holechlor Reation Dymamics and Chemical Reactivin: Oxford University Press: New York. U.S.A.. 1987.

4. (a) I3rumer. P. Shapiro, M. (hem. Iths /, ent 1986. 126.541: (b) Brumer. P. Shapiro. M. Imm. Rew Ifis (hen 1992. 43. 257; (c) Thu I. et al Science 1995. 370.77.

5. (a) Tannor. D. T.: Rice. S. A. J. (Thm. Phys. 1985. 83.5013 : (b) Koslott: R.: Rice. S. A.: Gaspard. P.: Tersgini. S.: Tanmor. D. $J$. (7trm. Phys. 1989. 139. 201: (c) Assion. A. at al Science 1998. 283. 919 .

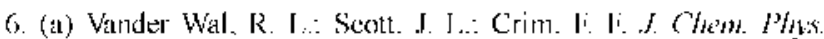
1990, 92, 8013; (b) Brown. S. S : B Berghout. II. T.: Crim. Ii. E. J. Chem. Phys. 1995. 102. 8440 (c) Crim. F. F. J. Phys. (7hm. 1996. 100.12725

7. Atomic and Holecth Beom Hethods: Scoles. G. Eds. Oxtord University Press: New York. UJ.S.A. 1988.

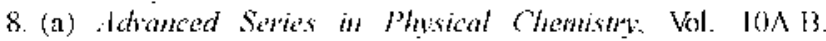
Photoinization and Photodetachment, Ng. C.-Y.. Fids.: World Scientitic: River Edgc. U.S.A. 2000: (b) Hiley Sures in Iom (Themistry and Plysics. High Resolution Lase Photoionization and Photolectron Studies. Powis. I.: Bacr. T.: No. C. Y.. Eds.: Wiley: New York, IJS.A. 1995: (c) I elokhow. V S. Iaser Fhotoionzation Spectroxcopy. Academic: Orlando. U.S.A. 1987: (d)

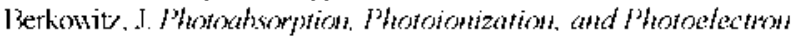
Spectroscopy. Academie: New York. U.S.A.. 1979.

9. (a) Bocsl. U.: Neusscr. H. I.: Schlag. E. W. Z. Katurforsch At 1978. 33. 1546: (b) Zandec. L.: Bernstein. R. B.: Liclitin. D. A.J. Chent Iths. 1978,69, 3427. 
10. (a) Brehm. B.: von Puttkamer. E. Z. Katurforsch. Feil 1967. 122. 8. (b) Cias Phase lon Chemishy l3owers, M. I. Jids.: Academic: New York. U.S.A. 1976), Wol. 1. Chap. 5; (c) 1)annacher. J. Org. Hass Spectrom 1984. 19. 253: (d) Haer 1. Anme. Ren: Phys. Chem. 1989. 40. 637: (c) Hsu. C.-W.: Lu. K. T.: Evans. M.: Chen. Y. J: Ng. C.-Y.: Heimann. P. J. C.htm. Phys. 1996. 105. 3950.

11. (a) Munson. M. S. B.: Ficld. F. H. J. in. (Them. Soc. 1966. 89. 2621: (b) l lajison. A. (i. Chemacal Ionization Mass Spectronetny. C.RC: IBoca Raton, U.S.A. 1983.

12. (a) Schlag. 1: W. ZENE Spectroscopv. Caunbridge University PTess: Cambridge. U.K. 1998: (b) Müller-Dethlets. K.: Sander. M: Schlag. E. W. C/rm. Phos, Lett. 1984. 112. 291.

13. Reiser. G.: Halvenicht. W: Müller-Dethletts. K.: Schlag. E. W. Chem. Phws. Lett 1988.152 .119

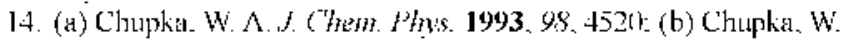
A.J. Chem. Plns. 1993. 99. 5800 . (c) Jiven. U.: J3en-Nun. M.: Levine. R. D. Chm. Phls. Lath 1993. 210. 416.

15. Zhu. L.: Johnson. P.J. Chom. Phns 1991. 9f. 5769.

16. (a) Park. S. T.: Kim. S. K.: Kim. M. S Nanme 2M12. 415. 306: (b) Park, S. 1.: Kim. M. S. J (Chem. Phs 2002, I77. 124: (c) Park. S. l': Kim. M. S. J. (Them. Hhs (subuilted) (d) Park. S. T.: Kim. M. S. J. Am Chm Sor 2002. 124.7614

17. (a) Park. S. T.: Kim. S. K.: Kim. M. S. J. Chem. Phns. 2001. H4. 5568: (b) Park. S. T.: Kim. S. K.: Kim. M. S. J. Chem. Phws. 2001. 115. 2492

18. (a) I Tilber (i.: I.ago, A.: Wallemslein. R. J. Opt Soc. Im. B 1987. 4.1753: (b) Marangos, I. P.: Shen, N.: Ma, I .: I Jutchinson. M. II. R.: Connerade, J. P.J. Opt. Soc. Am. B 1990. 7. 1254.

19. Mever. S. A.: Faris. G. W. Opt Lell 1998. 23. 204.

20. Nir. E.: Hunziker. H. E.: De Vris. M. S. And. Chrm. 1999. 71. 1674 .

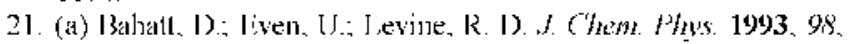
1744: (b) Soherer. W. G.: Selole II. J, : Schlag. Ii. W: I evine. R. 1). Plos Rer: Leth 1994, 72, 1435; (c) Jiven. IJ.: J evine R. I): Bcrsolnt. R. J. Plus Chem. 1994. 98. 3472.

22. Held. A.: Baranov: L. Y.: Selzlc. H. L.: Schlag. E. W. Z. Nantrforscth. i 1993. 48.1256 .
23. Bordas. C.: Bretet. P. F.: Broyer. M.: Chevaleyre. T.: Labastic. P.: Perrol. J. P. Phws. Rev Lett 1988.60.917.

24. (a) Merkt, 1.: 7are R. N. $J$. Chem. Iths 1994. 101, 3495: (b) Merkt. F.: Xu. H.: /are. R. N. J. (hom Phos, 1996. 104, 950; (c) Merkt. F. Amm. Ru Phos. ( $/ \mathrm{km}$ 1997. 48.675.

25. (a) Vrakking. M. J. J.: Lec. Y. T. J. Chum. Phws. 1995. 102.8818: (b) Vrakking. M. T. J.: Le. Y. T. f. (hem. Ihis 1995. 102.8833: (c) Vrakking. M. J. J : lischer, I.: Villeneuve I). M.; Stolow. A. J.

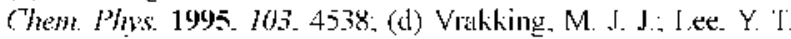
Phos Ror A 1995 5J R894: (e) Vrakking. M. J. I. J (Them. Phos. 1996. 105. 7336: (f) Vrakking. M. I. T. Phil. Trams. R. Soc. London Str.t 1997. 355. 1693 .

26. (a) Scherzer. W. G.: Selzle. H. L.: Schlag. E. W. \% Notuforsch . 1 1993. 48, 1256; (b) Nemeth. G. I.: Ungar, 11: Yeretrian, ( $\therefore$

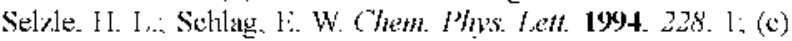
Scherzer. W. (j.: Selyle II I . Schlag L: W. I evine, R I) Plns. Rev: Left. 1994. 72. 1435: (d) Alt. C.: Seherzer. W. G.: Sclzle. H. L.: Schlag. E. W, (7hm. Plns. Lett. 1995. 240. 457: (c) Ramacle. F.: Levinc. R. D. J. (Them. I/hs 1996. 104. 1399: (1) Held. A.: Selzle II. I, : Schlag. H. W. J Ihws (7hem. 1996, 100, 15314: (g)

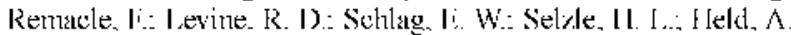
J. Phws (Them. 1996. 100. 15320) (h) Ileld. A: Baranow I. Y.; Sclzlc. H. L.: Schlag. E. W. J. (Kem. Plns. 1997. 106. 6848.

27. (a) Baranov. L. Y.: Held. A.: Selzle. H. L.: Schlag. E. W. Chem. Thus tett 1998. 291.311: (b) Held. A.: Baranov. L. Y.: Selzle. H.

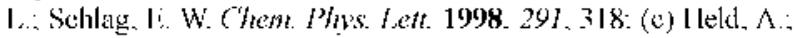
Aigner. U.: Baranov, I. Y.: Selzle. I. I..: Schlag. F. W. Chen. Phus. Lett 1999. 299. II () (d) Aigner, U.: I3aramow. I. Y.: Sel\%le. H. L.: Schlag. E. W. J. Electr: Spectrose: Relat. Phen. 2000. /12. 175.

28. Procter. S. R.: Webb. M. J.: Sottley. T. P. I Iaradar Iiscuss. 20 (Mo. 15. 277: (b) Solley, I. P.: Reduall, R. I. I. Chem. Phys 2000. II2. 7942 .

29. (a) Iones, R. R.: l'u. P. Gallagher T. F. J. Chem. Phws 1997. 106. 3578: (b) Bcllomo. P.: Farrelly: D.: Uzcr. T. J. ( $/ \mathrm{km}$. Phs. 1998. 108. 402: (c) Murgu. E.: Martin. J. D. D.: Gallagher. T. F. J. Chem. Hhis 2000. 113. 1321. 